\title{
STK17B promotes the progression of ovarian cancer
}

\author{
Liping Jiang ${ }^{1,2 \#}$, Jinhua Zhou ${ }^{1 \#}$, Shaojie Zhao ${ }^{2}$, Xuzhen Wang ${ }^{3}$, Youguo Chen ${ }^{1}$ \\ ${ }^{1}$ Department of Obstetrics and Gynecology, The First Affiliated Hospital of Soochow University, Suzhou, China; ${ }^{2}$ Department of Gynecology, \\ The Affiliated Wuxi Maternity and Child Health Care Hospital of Nanjing Medical University, Wuxi, China; ${ }^{3}$ Department of Breast Surgery, The \\ Affiliated Wuxi Maternity and Child Health Care Hospital of Nanjing Medical University, Wuxi, China \\ Contributions: (I) Conception and design: Y Chen; (II) Administrative support: J Zhou; (III) Provision of study materials or patients: Y Chen, S \\ Zhao; (IV) Collection and assembly of data: L Jiang; (V) Data analysis and interpretation: X Wang; (VI) Manuscript writing: All authors; (VII) Final \\ approval of manuscript: All authors. \\ \#These authors contributed equally to this work. \\ Correspondence to: Youguo Chen. Department of Obstetrics and Gynecology, The First Affiliated Hospital of Soochow University, 188 Shizi Road, \\ Suzhou 215006, China. Email: chenyouguo@suda.edu.cn.
}

Background: Protein kinase is increasingly receiving widespread attention because of its role in the tumor progression. Serine/threonine protein kinase (STK) is an important family involved in the development of a variety of cancers. Many studies have shown that serine/threonine kinase 17B (STK17B) is highly expressed in a variety of malignant tumors and participate in proliferation and metastasis. However, the exact function of STK17B remains uncertain in ovarian cancer. Our study aims to investigate whether STK17B plays a role in the occurrence and development of epithelial ovarian cancer.

Methods: We employed quantitative reverse transcription polymerase chain reaction to detect the relative expression of STK17B in ovarian cancer tissues. STK17B was down-regulated and up-regulated in ovarian cancer cell lines by small interfering RNA and overexpressed plasmid, respectively. The effects of STK17B on proliferation, invasion and migration of ovarian cancer cells in vitro were analyzed by CCK- 8 test, Transwell test, scratch test and EDU test. The tumorigenicity of subcutaneous xenograft tumor in nude mice to study the role of STK17B in tumorigenesis in vivo. Western Blotting analysis revealed that STK17B and EMT.

Results: STK17B expression was significantly increased in ovarian cancer tissues. The STK17B silencing suppressed cell progression, while the overexpression of STK17B promoted progression in vivo or in vitro. Western bolt showed that STK17B increased the invasion and migration of ovarian cancer cell by promoting the EMT process.

Conclusions: STK17B was highly expressed in epithelial ovarian cancer tissues and increased the proliferation, invasion and migration of ovarian cancer cells by promoting EMT process.

Keywords: STK17B; ovarian cancer; epithelial mesenchymal transition (EMT)

Submitted Dec 30, 2020. Accepted for publication Mar 03, 2021.

doi: $10.21037 /$ atm-21-601

View this article at: http://dx.doi.org/10.21037/atm-21-601

\section{Introduction}

Ovarian cancer has the highest mortality rate of all female reproductive system cancers (1-4). Due to its insidious onset, most patients are diagnosed at an advanced stage. Patients with advanced ovarian cancer often have extensive intraperitoneal metastasis that cannot be treated by surgery, resulting in tumor recurrence and death (5-8). Therefore, the aim of the present study was to elucidate the mechanism of ovarian cancer in order to determine the most effective therapy for its treatment.

Death-associated protein apoptotic kinase 2 (DRAK2); also known as serine/threonine kinase 17B (STK17B) is a threonine/serine kinase, a member of the family of death-related protein kinases, and a positive regulator of apoptosis (9). The MYB (v-myb avian myeloblastosis 
Table 1 Sequences of primers and siRNAs

\begin{tabular}{ll}
\hline Gene & Sequences \\
\hline PCR primer set & \\
STK17B & Forward: 5'-GCCTGTGTTTACCTGAGTTGG-3'; reverse: 5'-TGTCCCCGAGAGGGTATATGC-3' \\
GAPDH & Forward: 5'-CATCTCTGCCCCCTCTGCTGA-3'; reverse: 5'-GGATGACCTTGCCCACAGCCT-3' \\
siRNA set & \\
Si-STK17B-1 & Sense: 5'-GCCUGUGUUUACCUGAGUUTT-3'; antisense: 5'-AACUCAGGUAACACAGGCTT-3' \\
si-STK17B-2 & Sense: 5'-GCUACAGCAGUGGGACUUUTT-3'; antisense: 5'-AAAGUCCCACUGCUGUAGCTT-3' \\
\hline
\end{tabular}

viral) oncogene represses DRAK2 expression to suppress apoptosis in acute myeloid leukemia cells (10). The downregulation of STK17B in TRAIL-R1 mAb-sensitive cells upregulates Bcl-2 expression and restrains TRAIL-R1 mAb-induced apoptosis (11). DRAK2 is located a $2 \mathrm{q} 32.3$ deletion that was discovered by Sanjo et al. in 1998 (12). In recent years, STK17B has been found to be significantly upregulated in a variety of malignant tumors. For example, STK17B promotes carcinogenesis and metastasis in hepatocellular carcinoma (13). The aberrant expression of DRAK2 promotes tumorigenic potential by downregulating transforming growth factor- $\beta 1$ activity (14). STK17B could be a potential prognostic marker and therapeutic target in multiple myeloma patients (15).

Studies have found that epithelial mesenchymal transition (EMT) is involved in the development of tumors and promotes the migration of cancer cells (16-18).

In ovarian cancer, STK17B expression is significantly increased. Western bolt showed that STK17B increased the invasion and migration of ovarian cancer cell by promoting the EMT process. We present the following article in accordance with the ARRIVE reporting checklist (available at http://dx.doi.org/10.21037/atm-21-601).

\section{Methods}

\section{Tissue sample}

A total of 35 pairs of ovarian cancer samples were obtained from patients who had undergone ovariectomy. The study was approved by the Ethical Committee of The Affiliated Wuxi Maternity and Child Health Care Hospital of Nanjing Medical University (No. 2014-06-0120-06). All patients provided signed informed consent. The study was conducted in accordance with the Declaration of Helsinki (as revised in 2013).

\section{Cell line and culture}

SKOV3 and OV8 were obtained from the Institute of Cell Biology, Chinese Academy of Sciences in Shanghai. They were incubated in RPMI-1640 with $100 \mu \mathrm{g} / \mathrm{mL}$ streptomycin, $100 \mathrm{U} / \mathrm{mL}$ penicillin (Hyclone, Logan, UT, USA) and $10 \%$ fetal bovine serum (FBS). All cells were maintained at $37^{\circ} \mathrm{C}$ in $5 \% \mathrm{CO}_{2}$ in an incubator.

\section{Transfection}

si-STK17B-1 sense (5'-GCCUGUGUUUACCUGAGU UTT-3'), antisense 5'-AACUCAGGUAAACACAGGC T-3'; si-STK17B-2 sense (5'-GCUACAGCAGUGGGA UUUTT-3'), antisense 5'-AAAGUCCCACUGCUG AGCTT-3') (as shown in Table 1).

Gene Pharma (Suzhou, China) and Fubio (Suzhou, China) provided the negative control siRNA and si-RNA (si-STK17B), pcDNA3.1-STK17B, and pcDNA3.1-NC plasmids. Cells were harvested after $>24 \mathrm{~h}$ transfection in OV8 and SKOV3 cells using Lipofectamine 3000 (Invitrogen, USA).

\section{Quantitative reverse transcription polymerase chain reaction ( $q R T-P C R)$}

Total RNA was obtained by Trizol reagent (Invitrogen, USA), according to the manufacturer's instructions. STK17B primers were as follows: forward: 5'-GCC GTGTTTACCTGAGTTGG-3'and reverse: 5'-TG CCCCGAGAGGGTATATGC-3' (as shown in Table 1). The PrimeScript RT Reagent Kit with gDNA Eraser (Takara) was used to synthesize cDNA from whole RNA. STK17B expression was detected by qRT-PCR on the CFX96 sequence detection system (Bio-Rad) using SYBR Premix Ex Taq II (Takara). GAPDH and U6 small nuclear 
RNA were used as endogenous controls.

\section{Cell proliferation assay}

Ovarian cancer cell proliferation was assessed by Cell Counting Kit-8 (CCK-8) (Beyotime, Shanghai, China) and 5-ethynyl-20-deoxyuridine (EdU; Beyotime, China). Cells were inoculated in 96-well plates after transfection. At $0,24,48$, and $72 \mathrm{~h}, 10 \mu \mathrm{L}$ CCK-8 solution was added to each well. Cells were then cultured for a further 1-2 h. The optical density of the cells at a 450 -nm wavelength was measured. The EdU trial was played out according to the introduction.

\section{Migration assay}

Ovarian cancer cell migration was evaluated by the wound healing assay. Cells were cultivated in 6-well plates after $48 \mathrm{~h}$ of transfection with STK17B siRNA. 90\% confluence was obtained before transfection. A clean line in the middle of 6-well plates by $200-\mu \mathrm{L}$ sterilization pet tips, then washed the floating cells with PBS. Finally, the travel distances were taken pictures at 0 and $24 \mathrm{~h}$ in order to estimate the ability of cell wound healing.

\section{Transwell assay}

Cell invasion was detected by transwell chambers and Matrigel (Corning, USA). $100 \mu \mathrm{L}\left(5 \times 10^{4}\right)$ Serum-free medium contained cells were added into the upper chamber coated with Matrigel, and $500 \mu \mathrm{L}$ Dulbecco's modified Eagle's medium containing 20\% FBS was added to the bottom chamber. After $48 \mathrm{~h}$, cells in the upper chamber were removed, and cells in the lower membrane were stained with $0.1 \%$ crystal violet. Invaded cells were counted under a microscope.

\section{Western blot}

RIPA Lysis Buffer (Beyotime, China) with protease inhibitors was used to lyse cells for total protein extraction. After denaturation, the protein samples were separated with $10 \%$ sodium dodecylsulfate-polyacrylamide gel electrophoresis and transferred to a polyvinylidene difluoride membrane (Millipore, USA). After being blocked with $5 \%$ skim milk, the membrane, together with the primary antibodies, including STK17B, E-Cadherin, N-Cadherin, Vimentin, Twist, Slug, Snail., were incubated at $4{ }^{\circ} \mathrm{C}$ for
14-16 h. Above primary antibodies were then rinsed 3 times with TBST and incubated with anti-mouse or anti-rabbit horseradish peroxidase-coupled secondary antibodies for $1 \mathrm{~h}$ at room temperature. They were then rinsed 3 times with TBST. An electrochemical luminescence kit (Beyotime, China) was used to visualize the protein bands.

\section{Animal experiments}

In the present study, we used 5-6-week-old, weight 18-22 g, female BALB/c nude mice to research xenograft in vivo. OV8-LV-shNC and OV8-LV-shSTK17B cells $\left(5 \times 10^{6}\right)$ were injected into each mouse. Tumor growth was detected every 5 days. Subcutaneous tumor weights were detected after the mice were killed. Experiments were performed under a project license (GB/T 35,892-2018) granted by the Ethics Committee of Soochow University, in compliance with "Laboratory animal—Guideline for ethical review of animal welfare" for the care and use of animals.

\section{Statistical analysis}

Every assay was executed in triplicate. $T$-test or $\chi^{2}$-test was used to analyze every experimental assay. $\mathrm{P}<0.05$ was considered to be statistically significant. SPSS version 20.0 (IBM, Armonk, NY, USA) software was used for the statistical analyses.

\section{Results}

\section{Upregulated expression of STK17B in ovarian cancer}

STK17B expression was increased 2.429-folds in the carcinoma specimens $(\mathrm{P}<0.05)$ (Figure $1 A, B)$. Our findings indicated that STK17B could act as the oncogene in ovarian cancer.

We searched the STK17B expression using the Lu database and The Cancer Genome Atlas (TCGA) (Figure 1C,D). STK17B expression was found to be increased in ovarian carcinoma.

\section{STK17B acts as the oncogene in ovarian cancer, and knockdown of STK17B restrains cell progression}

STK17B expression decreased $(\mathrm{P}<0.01)$ in SKOV3 and decreased $(\mathrm{P}<0.01)$ in OV8 (Figure $2 A)$. Knockdown of STK17B suppressed cell proliferation in ovarian cancer cells $(\mathrm{P}<0.01)$ (Figure 2B). EdU-positive cells in the si-STK17B 

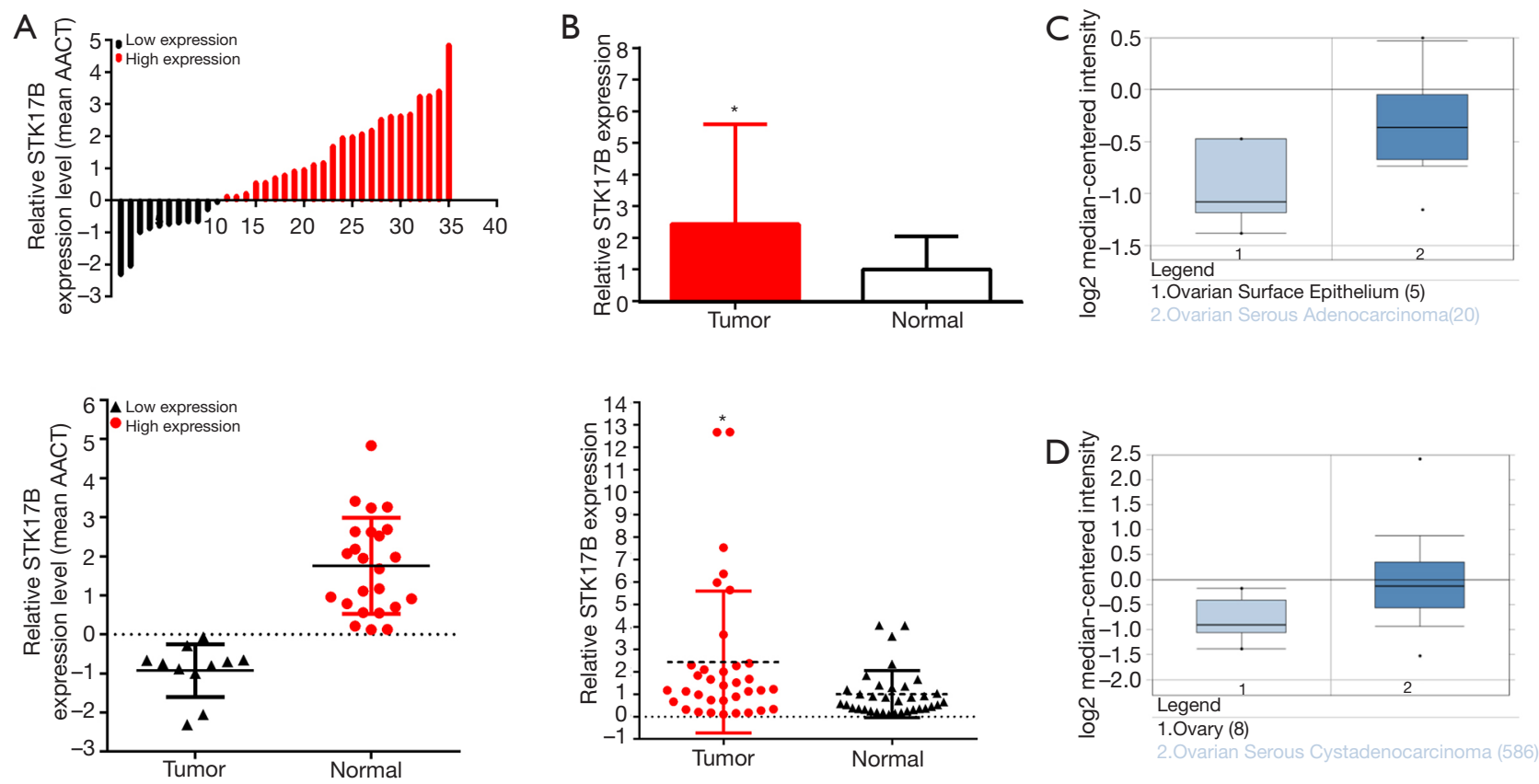

Figure 1 Expression of STK17B in ovarian cancer. Relative expression patterns of STK17B in ovarian cancer tissues (A) and normal tissues (B). Expression characteristics of STK17B was detected in the Lu database (C) and The Cancer Genome Atlas (D). * $\mathrm{P}<0.05$.

group decreased in SKOV3 $(\mathrm{P}<0.001)$ and OV8 $(\mathrm{P}<0.001)$ (Figure 2C), further demonstrating that STK17B promotes cell proliferation in ovarian cell lines.

The relative migration ratio in the si-STK17B group decreased $(\mathrm{P}<0.01$, si-STK17B-1 and $\mathrm{P}<0.05$, si-STK17B-2) in SKOV3 (Figure 2D). The relative migration ratio in the si-STK17B group decreased $(\mathrm{P}<0.001$, si-STK17B-1 and $\mathrm{P}<0.01$, si-STK17B-2) in OV8 (Figure 2D). In the transwell assay, the relative migration ratio decreased $(\mathrm{P}<0.001$, siSTK17B-1 and $\mathrm{P}<0.01$, si-STK17B-2) in SKOV3 and $(\mathrm{P}<0.0001$, si-STK17B-1 and $\mathrm{P}<0.001$, si-STK17B-2) in OV8 (Figure 2E). The relative invasion ratio in the si-STK17B group decreased $(\mathrm{P}<0.001)$ in SKOV3 and $(\mathrm{P}<0.01$, si-STK17B-1 and $\mathrm{P}<0.001$, si-STK17B-2) in OV8 (Figure $2 F$ ), further demonstrating that knockdown of STK17B suppressed ovarian cancer cell migration and invasion.

\section{Overexpression of STK17B promotes cell progression}

STK17B expression was upregulated 4.224-fold $(\mathrm{P}<0.01)$ in SKOV3 and 5.653-fold $(\mathrm{P}<0.001)$ in OV8 (Figure $3 A)$. The overexpression of STK17B accelerated cell proliferation in ovarian cancer cells $(\mathrm{P}<0.01)$ by CCK- 8 assay (Figure $3 B)$.

After the transfection of pcDNA3.1-STK17B, EdU- positive cells increased 3.354-fold in SKOV3 $(\mathrm{P}<0.001)$ and 2.303 -fold in OV8 $(\mathrm{P}<0.001)$ (Figure $3 C)$, further demonstrating that the overexpression of STK17B promoted cell proliferation in ovarian cancer cell lines.

The relative migration ratio in the pcDNA3.1-STK17B group was upregulated 2.229-fold in SKOV3 $(\mathrm{P}<0.001)$ and 2.217-fold in OV8 $(\mathrm{P}<0.001)$ by scratch assay, further demonstrating that the overexpression STK17B promoted ovarian migration (Figure 3D).

The relative migration ratio in the pcDNA3.1-STK17B group increased 2.339-fold in SKOV3 $(\mathrm{P}<0.01)$ and 2.183fold in OV8 $(\mathrm{P}<0.001)$ by transwell assay (Figure $3 E)$, demonstrating that the overexpression of STK17B promoted ovarian migration.

The relative invasion ratio in the pcDNA3.1-STK17B group was increased 2.425 -fold in SKOV3 $(\mathrm{P}<0.001)$ and upregulated 2.374 -fold in OV8 $(\mathrm{P}<0.001)$ by transwell assay (Figure $3 F$ ), demonstrating that the overexpression of STK17B promoted ovarian cancer migration.

\section{STK17B silencing suppresses ovarian cancer cell tumorigenicity}

Downregulation of STK17B suppressed ovarian cancer tumorigenicity in vivo (Figure $4 A, B, C, D, E$ ). Tumors 







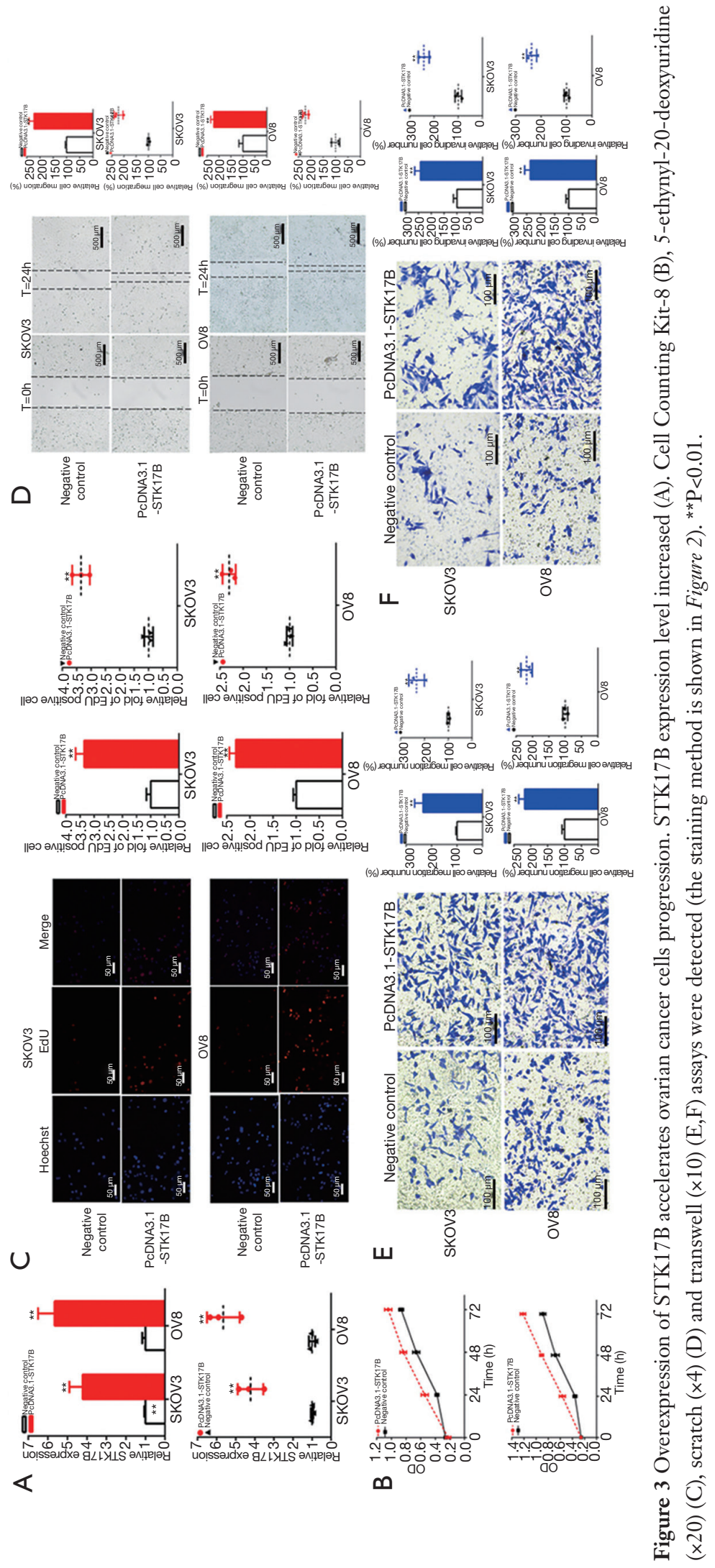


A

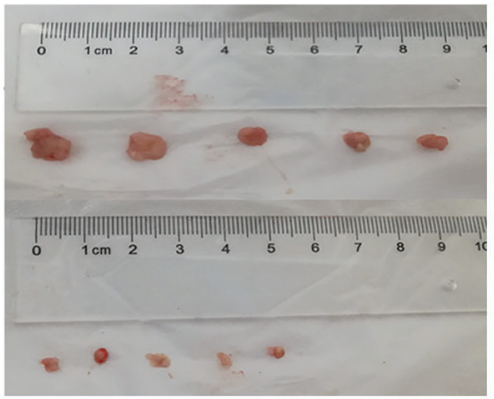

C

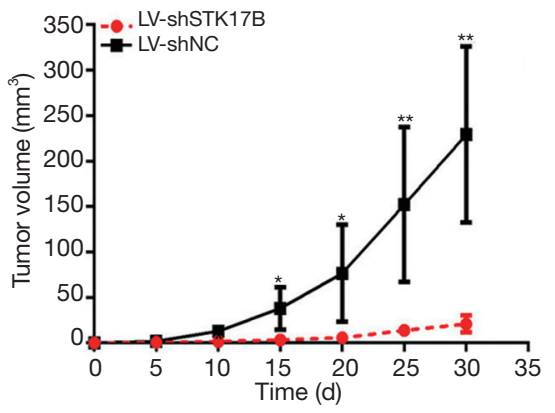

\section{B}

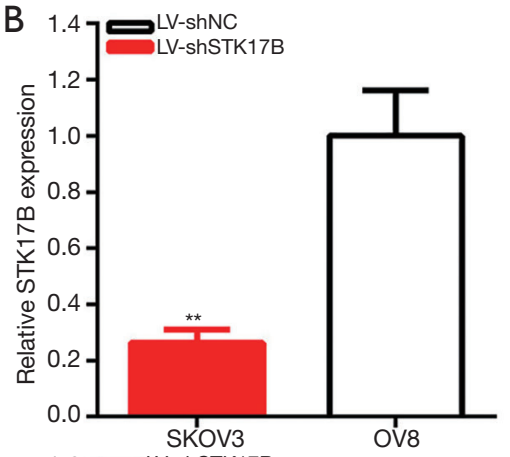

D ${ }^{1.6}{ }^{\mathrm{LV}-\mathrm{ShSTK} 17 \mathrm{~B}}$

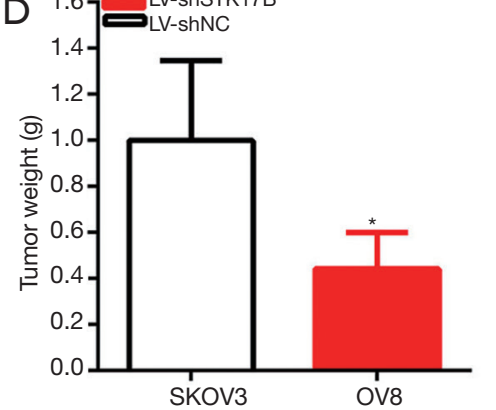

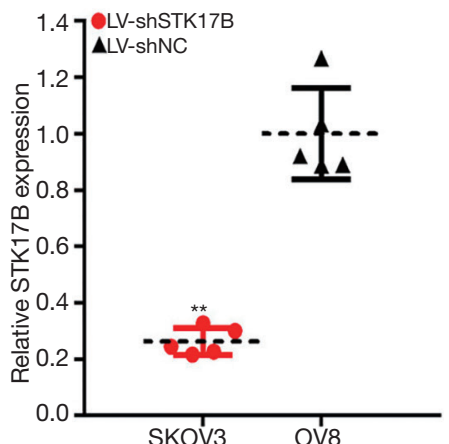

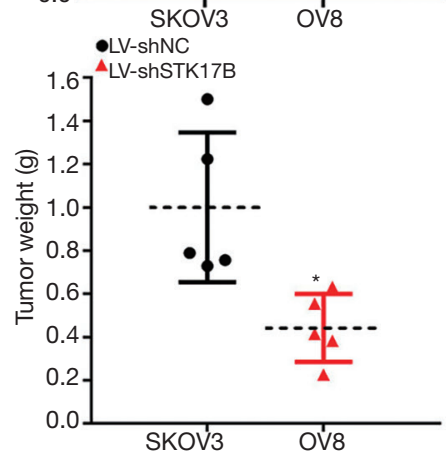

E

Skrta

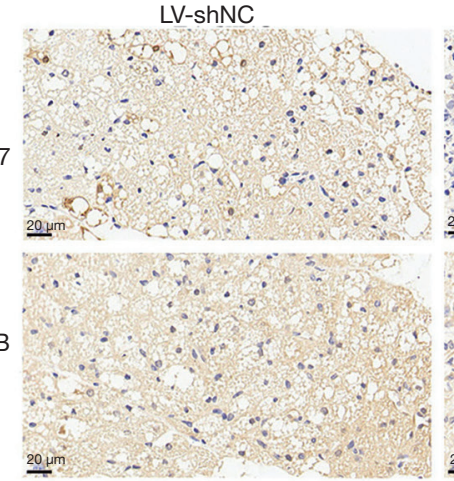

LV-shSTK17B

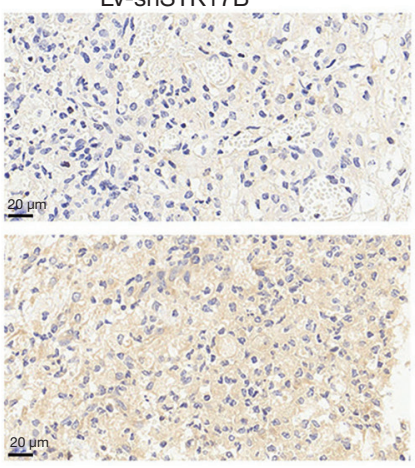

$\mathrm{F}$

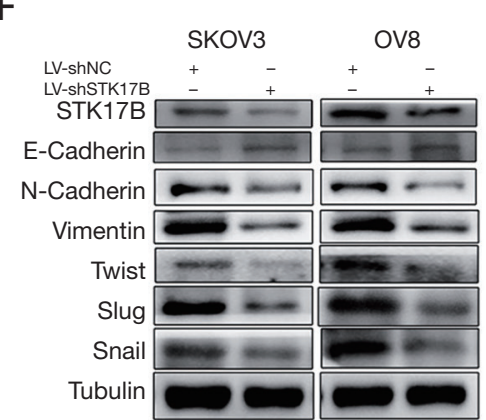

Figure 4 Knockdown of STK17B affects ovarian cancer cells tumorigenicity. Tumors are shown (A). STK17B expression level decreased (B). Tumor volume curve (C) and tumor weight were detected (D). Knockdown of STK17B downregulated Ki-67 and STK17B expression in vivo $(\times 40)$ by immunohistochemical analysis. (E). STK17B was involved in epithelial mesenchymal transition. Knockdown of STK17B decreased STK17B expression in ovarian cancer cells $(\mathrm{F}) .{ }^{*} \mathrm{P}<0.05,{ }^{* *} \mathrm{P}<0.01$.

collected from mice were shown in Figure 4A. Knockdown of STK17B was downregulated in vivo (Figure 4B). Tumor growth of the LV-NC treatment group was quicker than that of the LV-STK17B group (Figure 4C). Tumor weight was less in the LV-STK17B group (Figure 4D). STK17B downregulation decreased Ki-67 and STK17B expression (Figure 4E). These findings demonstrated that STK17B promotes ovarian cancer cell tumorigenicity in vivo.

\section{$S T K 17 B$ is involved in the EMT of ovarian cancer}

STK17B participated in the EMT of ovarian cancer cells (Figure 4F). EMT downstream genes were detect by Western blot. STK17B silencing restrained $\mathrm{N}$-cadherin and vimentin expression and increased E-cadherin expression in ovarian cancer cells; Twist, Slug, and Snail transcription factors decreased. These findings indicated that STK17B is involved in the EMT of ovarian cancer. 


\section{Discussion}

To date, the 5-year survival rate of advanced-stage ovarian cancer patients is still only about $40 \%$. Therefore, it is important to understand and control proliferation, invasion, and metastasis to improve the prognosis and survival rate of ovarian cancer patients.

The STK17B gene, also known as DRAK2, belongs to the DAPK (death-associated protein kinase) family $(19,20)$. STK17B is increased in basal-like and HER2-enriched breast cancer, while in a xenograft model, the absence of STK17B was found to inhibit tumor development and tumor growth (13). STK17B was highly expressed in cutaneous T-cell lymphoma (21), and was overexpressed in hepatocellular carcinoma (HCC) tissues (22). STK17B may promote tumor progression. However, the role of STK17B in various tumors is controversial. Some studies have found that STK17B could act as a tumor suppressor in leukemia and colorectal cancer $(10,22)$, and STK17B has been found to play a dual function in tumor progression (9,23). The role of STK17B is disease or cell dependent; previously published studies have indicated that STK17B is upregulated in most HCC tissues and can stimulate HCC cell proliferation and metastasis in vivo and in vitro $(12,21)$. STK17B could also inhibit the expression of epithelial cells by regulating the AKT/GSK-3 $\beta /$ Snail signaling pathway, increase the expression of interstitial cells ( $\mathrm{N}$-cadherin and vimentin), and promote EMT and tumorigenesis (12). It has been found that upregulation of STK17B is related to clinicopathological characteristics such as tumor size, TNM stage, and venous invasion (12). In the present study, we studied the role of STK17B and its underlying mechanism in ovarian cancer. STK17B expression was found to be upregulated in ovarian cancer tissues compared with normal ovarian tissue, which indicates that STK17B may be involved in cancer procession Our study in functional experiments have shown that STK17B silencing suppresses ovarian cancer cell proliferation, migration, and invasion, and the overexpression of STK17B promotes proliferation, migration, and invasion. We observed that STK17B was significantly tumorigenic compared with the control group in vivo. Therefore, we believe that STK17B acts as a significant tumor regulator and potential therapeutic target in ovarian cancer. Our results are consistent with the differential expression of ovarian cancer in the TCGA.

EMT is critical in the progression of metastasis in multiple cancers $(24,25)$; therefore, in the present study, we tried to verify whether STK17B participates in the EMT of ovarian cancer. In the Western blot results, we found that when STK17B was silenced, key proteins in EMT that modulates E-cadherin was upregulated and N-cadherin was downregulated. The Twist, Slug, and Snail transcription factors decreased in EMT. Therefore, we believe that STK17B may be involved in the progression of ovarian cancer by promoting EMT. However, we need to verify EMT signaling pathway in future studies.

\section{Conclusions}

STK17B is upregulated and promotes progression in ovarian cancer. STK17B may be involved in EMT progression in ovarian cancer.

\section{Acknowledgments}

The authors are grateful to the donors who participated in this program.

Funding: This work was supported by the National Natural Science Foundation of China (Nos. 81772773, 81302275, 81672560), Jiangsu Provincial Medical Youth Talent (Nos. QNRC2016753, QNRC2016752), Suzhou Clinical Key Technology Project (No. LCZX201705), Gusu Medical Youth Talent (No. GSWS2019034) and The Project of Jiangsu Provincial Maternal and Child Health Association (No. FYX201709).

\section{Footnote}

Reporting Checklist: The authors have completed the ARRIVE reporting checklist. Available at http://dx.doi. org/10.21037/atm-21-601

Data Sharing Statement: Available at http://dx.doi. org/10.21037/atm-21-601

Conflicts of Interest: All authors have completed the ICMJE uniform disclosure form (available at http://dx.doi. org/10.21037/atm-21-601). The authors have no conflicts of interest to declare.

Ethical Statement: The authors are accountable for all aspects of the work in ensuring that questions related to the accuracy or integrity of any part of the work are appropriately investigated and resolved. The study was approved by the Ethical Committee of The Affiliated Wuxi Maternity and Child Health Care Hospital of 
Nanjing Medical University (No. 2014-06-0120-06). All patients provided signed informed consent. The study was conducted in accordance with the Declaration of Helsinki (as revised in 2013). Experiments were performed under a project license (GB/T 35,892-2018) granted by the Ethics Committee of Soochow University, in compliance with "Laboratory animal—Guideline for ethical review of animal welfare" for the care and use of animals.

Open Access Statement: This is an Open Access article distributed in accordance with the Creative Commons Attribution-NonCommercial-NoDerivs 4.0 International License (CC BY-NC-ND 4.0), which permits the noncommercial replication and distribution of the article with the strict proviso that no changes or edits are made and the original work is properly cited (including links to both the formal publication through the relevant DOI and the license). See: https://creativecommons.org/licenses/by-nc-nd/4.0/.

\section{References}

1. Yang Y, Wei Q, Tang Y, et al. Loss of hnRNPA2B1 inhibits malignant capability and promotes apoptosis via downregulating Lin28B expression in ovarian cancer. Cancer Lett 2020;475:43-52.

2. Ma L, Sun Y, Li D, et al. Overexpressed ITGA2 contributes to paclitaxel resistance by ovarian cancer cells through the activation of the AKT/FoxO1 pathway. Aging (Albany NY) 2020;12:5336-51.

3. Wang Y, Qiao X, Yang X, et al. The role of a drug-loaded poly (lactic co-glycolic acid) (PLGA) copolymer stent in the treatment of ovarian cancer. Cancer Biol Med 2020;17:237-50.

4. Sheng R, Li X, Wang Z, et al. Circular RNAs and their emerging roles as diagnostic and prognostic biomarkers in ovarian cancer. Cancer Lett 2020;473:139-47.

5. Singh A, Gupta S, Sachan M. Epigenetic Biomarkers in the Management of Ovarian Cancer: Current Prospectives. Front Cell Dev Biol 2019;7:182.

6. Gao T, Zhang X, Zhao J, et al. SIK2 promotes reprogramming of glucose metabolism through $\mathrm{PI} 3 \mathrm{~K} /$ AKT/HIF-1 $\alpha$ pathway and Drp1-mediated mitochondrial fission in ovarian cancer. Cancer Lett 2020;469:89-101.

7. Zhang PF, Wu J, Luo JH, et al. SNHG22 overexpression indicates poor prognosis and induces chemotherapy resistance via the miR-2467/Gal-1 signaling pathway in epithelial ovarian carcinoma. Aging (Albany NY) 2019;11:8204-16.
8. Ali R, Alabdullah M, Alblihy A, et al. PARP1 blockade is synthetically lethal in XRCC1 deficient sporadic epithelial ovarian cancers. Cancer Lett 2020;469:124-33.

9. Farag AK, Roh EJ. Death-associated protein kinase (DAPK) family modulators: Current and future therapeutic outcomes. Med Res Rev 2019;39:349-85.

10. Ye P, Zhao L, Gonda TJ. The MYB oncogene can suppress apoptosis in acute myeloid leukemia cells by transcriptional repression of DRAK2 expression. Leuk Res 2013;37:595-601.

11. Sironen A, Uimari P, Nagy S, et al. Knobbed acrosome defect is associated with a region containing the genes STK17b and HECW2 on porcine chromosome 15. BMC Genomics 2010;11:699.

12. Sanjo H, Kawai T, Akira S. DRAKs, novel serine/threonine kinases related to death-associated protein kinase that trigger apoptosis. J Biol Chem 1998;273:29066-71.

13. Lan Y, Han J, Wang Y, et al. STK17B promotes carcinogenesis and metastasis via AKT/GSK-3 $3 /$ Snail signaling in hepatocellular carcinoma. Cell Death Dis 2018;9:236.

14. Yang KM, Kim W, Bae E, et al. DRAK2 participates in a negative feedback loop to control TGF- $\beta / S m a d s$ signaling by binding to type I TGF- $\beta$ receptor. Cell Rep 2012;2:1286-99.

15. Ni IB, Ching NC, Meng CK, et al. Translocation $\mathrm{t}(11 ; 14)$ (q13;q32) and genomic imbalances in multi-ethnic multiple myeloma patients: a Malaysian study. Hematol Rep 2012;4:e19.

16. Acloque H, Adams MS, Fishwick K, et al. Epithelialmesenchymal transitions: the importance of changing cell state in development and disease. J Clin Invest 2009;119:1438-49.

17. Xiao J, Gong Y, Chen Y, et al. IL-6 promotes epithelial-tomesenchymal transition of human peritoneal mesothelial cells possibly through the JAK2/STAT3 signaling pathway. Am J Physiol Renal Physiol 2017;313:F310-8.

18. Chang RM, Yang H, Fang F, et al. MicroRNA-331-3p promotes proliferation and metastasis of hepatocellular carcinoma by targeting $\mathrm{PH}$ domain and leucine-rich repeat protein phosphatase. Hepatology 2014;60:1251-63.

19. Boussios S, Abson C, Moschetta M, et al. Poly (ADPRibose) Polymerase Inhibitors: Talazoparib in Ovarian Cancer and Beyond. Drugs R D 2020;20:55-73.

20. Kuwahara H, Kamei J, Nakamura N, et al. The apoptosisinducing protein kinase DRAK2 is inhibited in a calciumdependent manner by the calcium-binding protein CHP. J Biochem 2003;134:245-50. 
21. Hartmann TB, Mattern E, Wiedemann N, et al. Identification of selectively expressed genes and antigens in CTCL. Exp Dermatol 2008;17:324-34.

22. Tomimaru Y, Koga H, Yano H, et al. Upregulation of T-cell factor-4 isoform-responsive target genes in hepatocellular carcinoma. Liver Int 2013;33:1100-12.

23. Doherty GA, Byrne SM, Austin SC, et al. Regulation of the apoptosis-inducing kinase DRAK2 by cyclooxygenase-2 in colorectal cancer. Br J Cancer 2009;101:483-91.

Cite this article as: Jiang L, Zhou J, Zhao S, Wang X, Chen Y. STK17B promotes the progression of ovarian cancer. Ann Transl Med 2021;9(6):475. doi: 10.21037/atm-21-601
24. Du B, Shim JS. Targeting Epithelial-36. Mesenchymal Transition (EMT) to Overcome Drug Resistance in Cancer. Molecules 2016;21:965.

25. Teeuwssen M, Fodde R. Wnt Signaling in Ovarian Cancer Stemness, EMT, and Therapy Resistance. J Clin Med 2019;8:1658.

(English Language Editor: R. Scott) 